\title{
Revealing the dilemma in COVID-19 pneumonia: use of the prone thorax CT imaging in differentiation of opacificities due to dependent zone and pneumonic consolidation
}

\author{
๑Enes Gürün1, ๑İsmail Akdulum², ФMelih Akyüz ${ }^{3}$ \\ 'İskilip Atıf Hoca State Hospital, Çorum, Turkey \\ ${ }^{2}$ Department of Radiology, Gazi University, Ankara, Turkey \\ ${ }^{3}$ Department of Diagnostic Radiology and Nuclear Medicine, Rush University Medical Center, Chicago, IL
}

Cite this article as: Gürün E, Akdulum İ, Akyüz M. Revealing the dilemma in COVID-19 pneumonia: use of the prone thorax CT imaging in differentiation of opacificities due to dependent zone and pneumonic consolidation. Anatolian Curr Med J 2021; 3(1): 78-80.

\begin{abstract}
In December 2019, a disease called coronavirus disease 2019 (COVID-19) caused by the novel coronavirus called severe acute respiratory syndrome coronavirus 2 (SARS-COV-2) emerged in Wuhan, China. The disease was declared as pandemic by the WHO on May 11, 2020. The gold standard diagnostic test is a real-time reverse transcriptase-polymerase chain reaction (RTPCR). Additionally, computed tomography (CT) could be a good alternative in certain clinical scenarios. A 62-year-old female patient was admitted to the emergency room with fever and joint pain for the last five days. She had a history of a contact with COVID-19 (+) patient. Thorax CT without contrast was performed in the prone position clearly revealed nodular infiltrations in the subpleural/peripheral lower lung zones in the right lung. A 73-year- old 73-year-old female patient was admitted to the emergency room with the complaints of tiredness and loss of taste for a few days. She had no history of a contact with COVID-19 (+) patients. Thorax CT without contrast was performed while the patient was in supine position. Diffuse nodular opacities in the lower regions of both lungs were barely identified. The combined throat and nose swab tests were positive in both patients. In conclusion, thorax CT in prone position is a very valuable methodin imaging of COVID-19 (+) patients; regarding differentiation of true COVID-19 pneumonia consolidations and dependent zone opacities. True COVID-19 pneumonia from dependent lung zones in the supine position.
\end{abstract}

Keywords: COVID-19, thorax CT in prone position, dependent zone opacity

\section{INTRODUCTION}

In December 2019, a disease called coronavirus disease 2019 (COVID-19) caused by the novel coronavirus called severe acute respiratory syndrome coronavirus 2 (SARS- COV-2) emerged in Wuhan, China. The disease was declared as pandemic by the WHO on May 11, 2020. On 5 September 2020 total case number was 26.468 .031 and mortality was 871.166 (1). Clinical symptoms include but not limited to fever, dry cough, sore throat, joint pain, headache, and dyspnea. The gold standard to diagnose is a real-time reverse transcriptase-polymerase chain reaction (RT-PCR). Additionally, computed tomography (CT) is also very useful in certain cases. Currently in the literature, common thorax findings are bilateral lower lobe predominant peripheral-subpleural ground glass nodular opacities, interlobular septal thickening. Anatomically, the most common areas of infiltration are the posterior segments of the lower lobes (2-4). Gravity-dependent atelectasis refers to a type of lung atelectasis caused by a

combination of reduced alveolar volume and increased perfusion in the dependent portions of the lungs. Due to the gravity, it usually has a subpleural distribution and base of the lung (5).

\section{CASE REPORT}

A 62-year-old female patient was admitted to the emergency room with fever and joint pain for the last five. The patient had chronic hypertension and a history of contact with COVID-19 (+) patient. There was no other significant finding in the physical examination and clinical history. Vitals were within normal limits and laboratory findings were as follows: C-reactive protein (CRP) 39.6 $\mathrm{mg} / \mathrm{L}$, lactate dehydrogenase (LDH) $243 \mathrm{U} / \mathrm{L}$, white blood cell count (WBC) $5.83 \times 10^{9} / \mathrm{L}$, hemoglobin count 12.6 $\mathrm{g} / \mathrm{dL}$, ferritin $191 \mathrm{ng} / \mathrm{Ml}$. The patient was isolated as a probable COVID-19 case. Thorax CT without contrast was 
performed with the patient in a prone position. Nodular infiltrations were found in subpleural/peripheral lower lung zones in the right lung (Figure 1). The combined throat/nose swab of the patient was positive for SARSCOV-2 one day later. The patient was treated as follows; 2x200 mg hydroxychloroquine, $1 \times 0.6 \mathrm{IU} / \mathrm{mL}$ enoxaparin, and 1x250 mg azithromycin as per the guidelines at that time. The patient had no additional findings during the clinical follow-up and was discharged after five days of hospitalization.

A 73-year-old female patient was admitted to the emergency room with tiredness and loss of taste for a few days. She had no history of contact with COVID- 19 (+) patients. There was no significant finding in physical examination. Vitals were stable. Laboratory findings were as follows: C-reactive protein (CRP) $8.8 \mathrm{mg} / \mathrm{L}$, lactate dehydrogenase (LDH) $268 \mathrm{U} / \mathrm{L}$, white blood cell (WBC) $3.35 \times 10^{9} / \mathrm{L}$, hemoglobin count $12.1 \mathrm{~g} / \mathrm{dL}$, ferritin $314.1 \mathrm{ng} /$ Ml. Thorax CT without contrast was performed with the patient in a supine position. Widespread nodular opacities were found in lower zones of both lungs. In posterior lung fields, nodular opacities were superimposed with the dependent zone opacities and hardly differentiated from each other (Figure 2). The combined throat/nose swab test was positive for SARS-COV-2 one day later. The patient was treated as follows; $2 \times 200 \mathrm{mg}$ hydroxychloroquine, 1x0.6 IU/mL enoxaparin, and azithromycin 1x250 mg as per the guidelines at that time. The patient had no additional findings during the clinical follow-up and was discharged after five days of hospitalization.

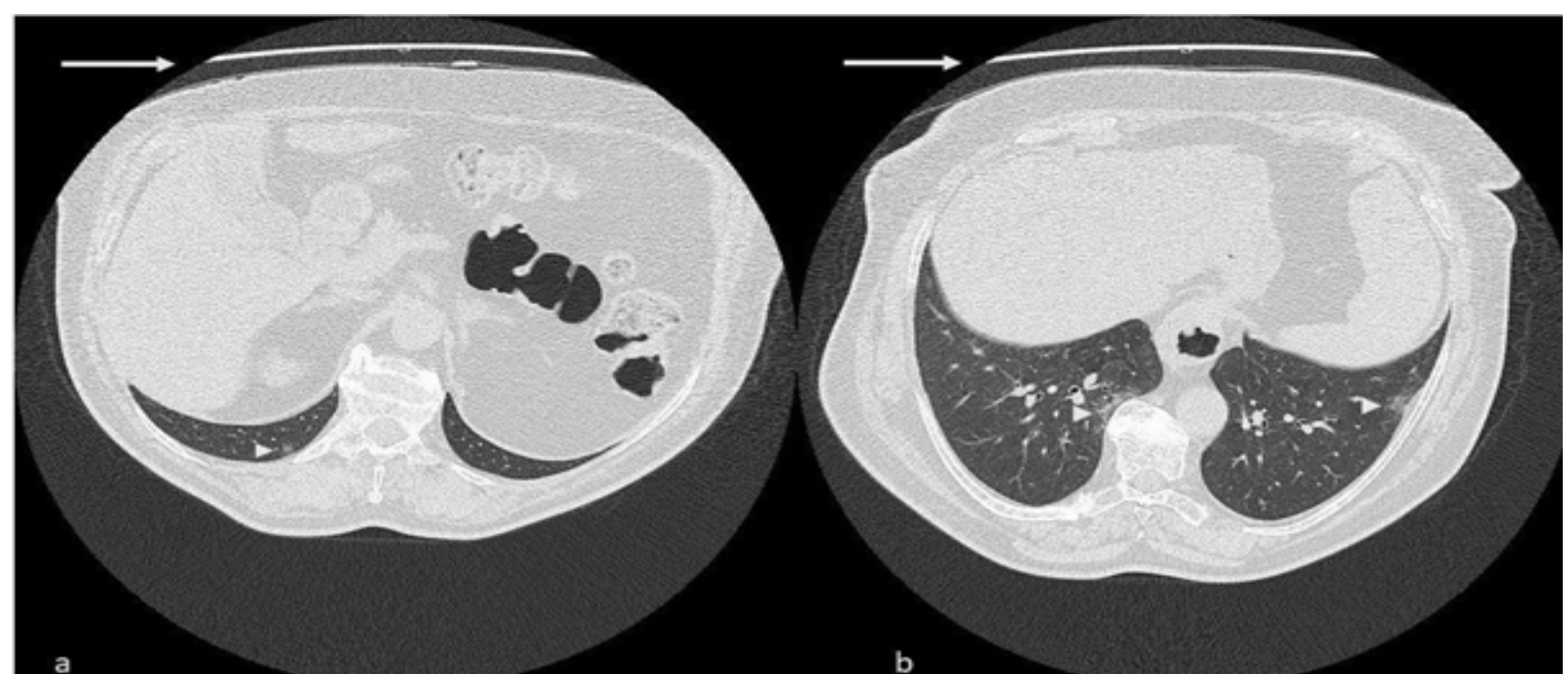

Figure 1. (a, b) 62-year-old female with COVID-19. Thorax CT in a prone position (CT table: arrow). Axial (a-b) CT images revealed subpleural ground-glass opacities seen in bilateral lower posterior lung zones (arrowhead).

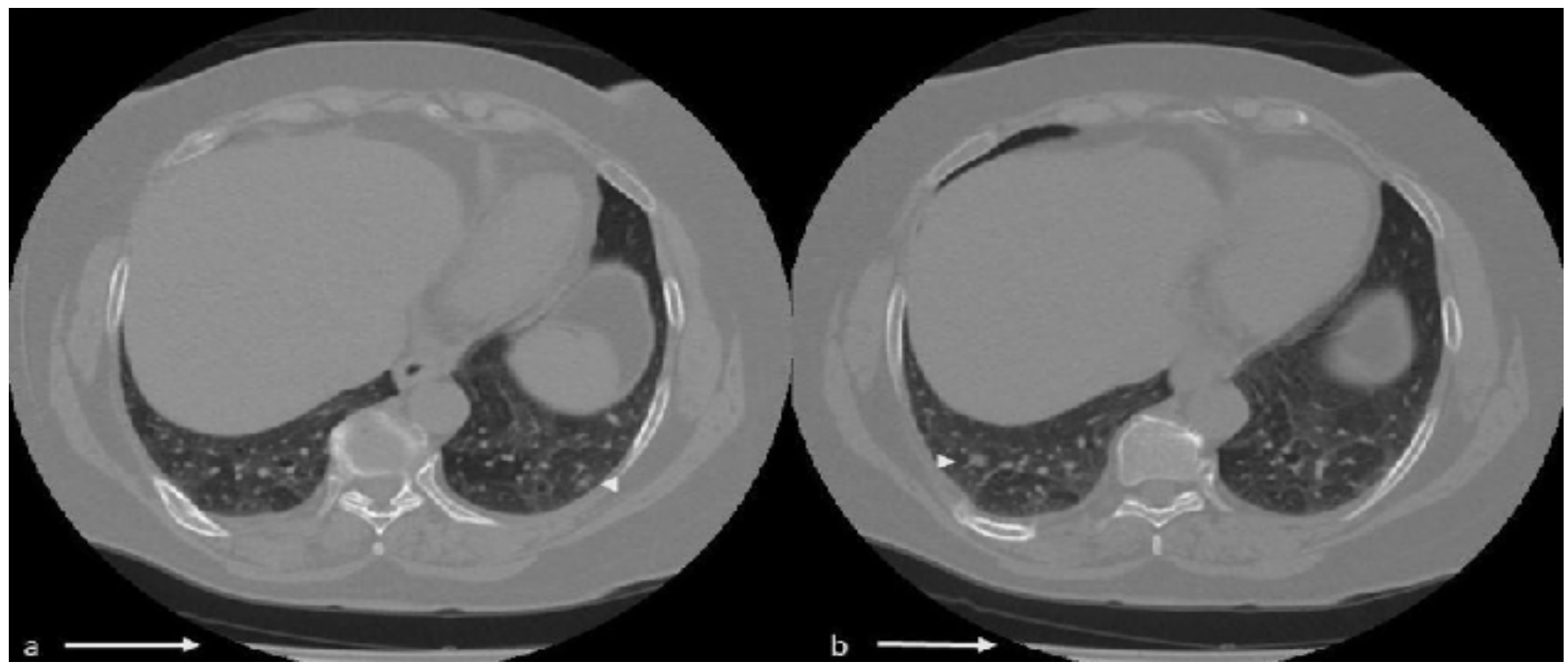

Figure 2. (a, b) 73-year-old female patient with COVID-19 infection. Thorax CT in a supine position (CT table: arrow). Axial (a-b) CT images revealed subpleural/peripheral nodular infiltrations which are hardly differentiated dependent zone opacities (arrowhead) 


\section{DISCUSSION}

Especially in elderly, gravity-dependent atelectasis and resultant opacities in the dependent zones in the posterior lung fields are commonly visualized when the thorax CT imaging is performed in a supine position. These findings might be confused with the COVID-19 pneumonia findings, and this confusion might result in misdiagnosis when the PCR testing is unavailable. For these reasons, we think that performing thorax $\mathrm{CT}$ in a prone position instead of a supine position might be more useful in the evaluation of the elderly patients with COVID-19 suspicion.

\section{CONCLUSION}

Although routine supine thorax $\mathrm{CT}$ is a highly valuable imaging method for evaluation of the COVID-19 pneumonia. Pneumonic infiltrates in lower lung fields might be confused with dependent zone atelectasis. Additionally, for patients suffering from miscellaneous diseases involving cardiovascular and respiratory systems dependent zones might be considered mistakenly as pneumonic infiltration that leads to improper clinical management and delays in treatment. We think it might be beneficial to have thorax $\mathrm{CT}$ imaging in a prone position instead of a supine position to better evaluate opacifications in the posterior lower lung fields, especially for the patients with respiratory and cardiac problems.

\section{ETHICAL DECLARATIONS}

Informed Consent: Written informed consent was obtained from all participants who participated in this study.

Referee Evaluation Process: Externally peer-reviewed.

Conflict of Interest Statement: The authors have no conflicts of interest to declare.

Financial Disclosure: The authors declared that this study has received no financial support.

Author Contributions: All of the authors declare that they have all participated in the design, execution, and analysis of the paper, and that they have approved the final version.

\section{REFERENCES}

1. Novel coronavirus (2019-nCoV) situation reports. Available from: https://www.who.int/emergencies/diseases/novelcoronavirus-2019/situation-reports.

2. Ai T, Yang Z, Hou H, et al. Correlation of chest CT and RT-PCR testing for coronavirus disease 2019 (COVID-19) in China: a report of 1014 cases. Radiology 2020; 296: E32-E40. doi:10.1148/ radiol.2020200642
3. Chung M, Bernheim A, Mei X, et al. CT imaging features of 2019 novel coronavirus (2019-nCoV). Radiology 2020; 295: 202-7.

4. Song F, Shi N, Shan F, et al. Emerging 2019 novel coronavirus (2019-nCoV) Pneumonia. Radiology. 2020; 295: 210-7.

5. Kashiwabara K, Kohshi S. Additional computed tomography scans in the prone position to distinguish early interstitial lung disease from dependent density on helical computed tomography screening patient characteristics. Respirology 2006; 11: 482-7. 